\title{
A class of $\varphi$-concave operators and applications
}

Yanbin Sang

"Correspondence: syb6662004@163.com

Department of Mathematics, North University of China, Taiyuan, Shanxi 030051, P.R. China

\section{Springer}

\begin{abstract}
In this paper, by means of the concept of $\varphi$-concave operators, which was introduced by Li and Liang (J. Systems Sci. Math. Sci. 14(4):355-360, 1994 (in Chinese)), we obtain some new existence and uniqueness theorems of a fixed point of mixed monotone operators with such concavity. Moreover, we apply the main theorem to a class of Hammerstein integral equations.
\end{abstract}

Keywords: cone and partial order; $\varphi$-concave operator; mixed monotone operator; fixed point

\section{Introduction}

It is well known that concave operators are a class of important operators that are extensively used in nonlinear differential and integral equations (see [1-12]). In [13], Krasnoselskii introduced in detail many important ideas and results about concave operators. A problem is that there are various concepts of concave operators, such as $u_{0}$-concave operators [5], ordered concave operators [1] and $\alpha$-concave operators [7], which is somewhat confusing. We should mention that Liang et al. [14] proved that both ordered concave operators and $\alpha$-concave operators are $u_{0}$-concave operators and gave necessary and sufficient conditions on which $u_{0}$-concave operators have a unique fixed point. Furthermore, Zhai et al. [11] considered an operator equation with general $\alpha$-concave or homogeneous operator, their results improved previous results (see Corollary 3.3 in [7]).

We note that Li and Liang [15] introduced the definition of $\varphi$-concave operator, which provided a general method to copy with such a class of operators together. Furthermore, $[8,9]$ extended the concept of $\varphi$-concave operator to $\phi$-concave $(-\psi)$ convex operators. In [12], Zhao weakened some conditions and strengthened the conclusions. Mixed monotone operators were introduced by Guo and Lakshmikantham [16] in 1987. Thereafter, many authors have focused on various existence (and uniqueness) theorems of fixed points for mixed monotone operators; for details, see $[8,9,13,16-33]$ and references therein. In [20], Bhaskar and Lakshmikantham established some coupled fixed point theorems for mixed monotone operators in partially ordered metric spaces and discussed the existence and uniqueness of a solution for a periodic boundary value problem. Instead of using a direct proof as in [20], Drici et al. [22] employed the notion of reflection operator and investigated fixed point theorems for mixed monotone operators by weakening the requirements in the contractive assumption and strengthening the metric space utilized with a partial order. Sintunavarat and Kumam [34] extended classical coupled fixed point theorems of Bhaskar and Lakshmikantham [20] to the coupled common fixed point theorems

○2013 Sang; licensee Springer. This is an Open Access article distributed under the terms of the Creative Commons Attribution License (http://creativecommons.org/licenses/by/2.0), which permits unrestricted use, distribution, and reproduction in any medium, provided the original work is properly cited. 
for mappings satisfying a new non-commuting condition. These theorems are generalizations of the results of [20]. Furthermore, Harjani et al. [25] improved the main results of [22] using the altering distance functions. Sintunavarat et al. [35] established some coupled fixed point theorems for a contraction mapping induced by the cone ball metric in partially ordered spaces and also discussed the condition claim of the uniqueness of a coupled fixed point. Very recently, Chandok et al. [21] were concerned with some coupled coincidence point theorems for a pair of mappings having a mixed $g$-monotone property in partially ordered G-metric spaces. Also, they presented a result on the existence and uniqueness of coupled common fixed points. In [19], the authors introduced the concept of $W$-compatible mappings. Based on this notion, a tripled coincidence point and a common tripled fixed point for mappings $F: X \times X \times X \rightarrow X$ and $g: X \rightarrow X$ were obtained, where $(X, d)$ is a cone metric space. We should point out that their results do not rely on the assumption of normality condition of the cone.

On the other hand, there is much attention paid to mixed monotone operators with certain concavity and convexity; for example, see [8, 9, 23, 24, 27, 28, 30-33]. In [32], Zhang and Wang modified the methods in [30, 31] to obtain some new existence and uniqueness results of a positive fixed point of mixed monotone operators. Recently, in [29], Zhai and Zhang presented a new fixed point theorem for a class of general mixed monotone operators, which extends the existing corresponding results. Moreover, they investigated some pleasant results of nonlinear eigenvalue problems with mixed monotone properties. Based on them, the local existence-uniqueness of positive solutions for nonlinear boundary value problems which include Neumann boundary value problems, three-point boundary value problems and elliptic boundary value problems for Lane-Emden-Fowler equations was proved.

In this paper, by means of the concept of $\varphi$-concave operator, some new theorems of fixed points of mixed monotone operators with such concavity are obtained. Our theorems unify and extend some previous results, then we apply these results in discussing a class of Hammerstein integral equations and give the new condition for determining the unique solution to this equation.

\section{Preliminaries}

Some definitions, notations and known results are from Refs. [2, 3, 23]. Let $E$ be a real Banach space. A nonempty convex closed set $P$ is called a cone if it satisfies the following conditions:

(i) $x \in P, \lambda \geq 0$ implies $\lambda x \in P$;

(ii) $x \in P$ and $-x \in P$ imply $x=\theta$, where $\theta$ denotes the zero element of $E$.

Let $E$ be partially ordered by a cone $P$ of $E$, i.e., $x \leq y$, if and only if $y-x \in P$ for any $x, y \in E$. Recall that the cone $P$ is said to be solid if the interior $P^{\circ}$ is nonempty, and $P$ is said to be normal if there exists a positive constant $M$ such that $\theta \leq x \leq y(x, y \in E)$ implies $\|x\| \leq M\|y\|$, where $M$ is the normal constant of $P$. Let $D \subset E$. An operator $A: D \times D \rightarrow E$ is said to be mixed monotone if $A(x, y)$ is nondecreasing in $x$ and nonincreasing in $y$, that is, for any $x, y \in D$,

$$
x_{1}, x_{2} \in D, \quad x_{1} \leq x_{2} \quad \Longrightarrow \quad A\left(x_{1}, y\right) \leq A\left(x_{2}, y\right)
$$


and

$$
y_{1}, y_{2} \in D, \quad y_{1} \leq y_{2} \quad \Longrightarrow \quad A\left(x, y_{1}\right) \geq A\left(x, y_{2}\right)
$$

An element $x^{*} \in D$ is called a fixed point of $A$ if it satisfies $A\left(x^{*}, x^{*}\right)=x^{*} . A: D \subset E \rightarrow E$ is said to be convex if for $x, y \in D$ with $x \leq y$ and each $t \in[0,1]$, we have

$$
A(t x+(1-t) y) \leq t A x+(1-t) A y
$$

$A$ is said to be concave if $-A$ is convex.

Let $h>\theta$, write $P_{h}=\{x \in E \mid \exists \lambda, \mu>0$, such that $\lambda h \leq x \leq \mu h\}$.

Let $e>\theta$. An operator $A: P \rightarrow P$ is said to be $e$-concave if it satisfies the following two conditions:

(i) $A$ is $e$-positive, i.e., $A(P-\{\theta\}) \subset P_{e}$;

(ii) $\forall x \in P_{e}, \forall 0<t<1, \exists \eta=\eta(t, x)>0$ such that

$$
A(t x) \geq(1+\eta) t A x
$$

where $\eta=\eta(t, x)$ is called the characteristic function of $A$.

The operator $T: P^{\circ} \rightarrow P^{\circ}$ is said to be $\alpha$-concave $(-\alpha$-convex $)(0 \leq \alpha<1)$ if

$$
T(t x) \geq t^{\alpha} T(x) \quad\left(T(t x) \leq t^{-\alpha} T(x)\right), \quad \forall x \in P^{\circ}, \forall t \in(0,1]
$$

Let $u_{0}, v_{0} \in E$ with $u_{0} \leq v_{0}$. Write

$$
\left[u_{0}, v_{0}\right]=\left\{x \in E \mid u_{0} \leq x \leq v_{0}\right\}
$$

where $\left[u_{0}, v_{0}\right]$ is called an ordering interval.

Definition 2.1 (see [25]) Let $A: P_{h} \rightarrow P_{h}$ be a $\varphi$-concave operator if there exists a function $\varphi:(0,1] \times P_{h} \rightarrow(0,1]$ such that $t \in(0,1)$ implies $t<\varphi(t, x)$, and $A$ satisfies the following condition:

$$
A(t x) \geq \varphi(t, x) A x, \quad 0<t \leq 1, x \in P_{h} .
$$

Definition 2.2 (see [2]) Let $\mathrm{S}$ be a bounded set of a real Banach space $E$. Let

$$
\alpha(S)=\inf \left\{\delta>0 \mid S=\bigcup_{i=1}^{m} S_{i} \text { with } \operatorname{diam}\left(S_{i}\right) \leq \delta, i=1,2, \ldots, m\right\}
$$

Clearly, $0 \leq \alpha(S)<\infty . \alpha(S)$ is called the Kuratowski measure of noncompactness.

Definition 2.3 (see $[36,37]$ ) Let $D \subset E, A: D \rightarrow E$ be an operator. Then $S$ is said to be a generalized condensing operator if for any $S \subset D, \alpha(S) \neq 0$ implies $\alpha(A(S))<\alpha(S)$.

Lemma 2.1 (see [2,37]) Let $S$, $T$ be bounded subsets of E. Then

(i) $\alpha(S)=0$ if and only if $S$ is relatively compact;

(ii) $S \subset T$ implies $\alpha(S) \leq \alpha(T)$; 
(iii) $\alpha(\bar{S})=\alpha(S)$;

(iv) $\alpha(S \cup T)=\max \{\alpha(S), \alpha(T)\}$;

(v) $\alpha(\operatorname{coS})=\alpha(S)$, where $\operatorname{coS}$ denotes the convex hull of $S$.

\section{Main results}

Theorem 3.1 Let $E$ be a real Banach space and $P$ be a normal cone of $E$. Let $u_{0}, v_{0} \in E$ with $u_{0} \leq v_{0}$ and $A:\left[u_{0}, v_{0}\right] \times P \rightarrow P$ be a mixed monotone operator. For fixed $v \in P$, $A(\cdot, v):\left[u_{0}, v_{0}\right] \rightarrow P$ is a $\varphi$-concave operator. Suppose that

(i) there exists a real positive number $r_{0}$ such that $u_{0} \geq r_{0} v_{0}$;

(ii) $u_{0}$ and $v_{0}$ are such that

$$
u_{0} \leq A\left(u_{0}, v_{0}\right), \quad A\left(v_{0}, u_{0}\right) \leq v_{0}
$$

(iii) there exists an element $w_{0} \in\left[u_{0}, v_{0}\right]$ such that

$$
\varphi(t, x) \geq \varphi\left(t, w_{0}\right), \quad \forall(t, x) \in(0,1) \times\left[u_{0}, v_{0}\right],
$$

and

$$
\lim _{s \rightarrow t^{-}} \varphi\left(s, w_{0}\right)>t, \quad \forall t \in(0,1)
$$

(iv) for fixed $u \in\left[u_{0}, v_{0}\right], \exists N>0$ such that

$$
A(u, \cdot): P \rightarrow P, \quad A\left(u, v_{1}\right)-A\left(u, v_{2}\right) \geq-N\left(v_{1}-v_{2}\right), \quad \forall v_{1} \geq v_{2}, v_{1}, v_{2} \in P .
$$

Then $A$ has exactly one fixed point $x^{*}$ in $\left[u_{0}, v_{0}\right]$.

Proof We divide the proof into three steps.

Step 1 . We prove that for any fixed $u \in\left[u_{0}, v_{0}\right], A(u, \cdot)$ has exactly one fixed point $T(u) \in$ $\left[A\left(u_{0}, v_{0}\right), A\left(v_{0}, u_{0}\right)\right]$ such that $A(u, T(u))=T(u)$. Our proof is the same as Theorem 2.1 in [32]. For completeness, we list it as follows.

For fixed $u \in\left[u_{0}, v_{0}\right]$, there exists $N>0$ such that $A(u, v)+N v$ is increasing in $v$. Let

$$
B(u, v)=\frac{A(u, v)+N v}{N+1},
$$

then $B(u, v)=v$ is equivalent to $A(u, v)=v$.

Let $x_{0}=A\left(u_{0}, v_{0}\right), y_{0}=A\left(v_{0}, u_{0}\right)$ and $x_{n+1}=B\left(u, x_{n}\right), y_{n+1}=B\left(u, y_{n}\right)$. By $u_{0} \leq u \leq v_{0}, x_{0}=$ $A\left(u_{0}, v_{0}\right) \leq v_{0}, u_{0} \leq v_{0}$ and $y_{0}=A\left(v_{0}, u_{0}\right) \geq u_{0}$, we have

$$
\begin{aligned}
& A\left(u, x_{0}\right) \geq A\left(u_{0}, v_{0}\right)=x_{0}, \\
& A\left(u, y_{0}\right) \leq A\left(v_{0}, u_{0}\right)=y_{0},
\end{aligned}
$$

thus

$$
\begin{aligned}
& x_{1}=B\left(u, x_{0}\right)=\frac{A\left(u, x_{0}\right)+N x_{0}}{N+1} \geq \frac{x_{0}+N x_{0}}{N+1}=x_{0}, \\
& y_{1}=B\left(u, y_{0}\right)=\frac{A\left(u, y_{0}\right)+N y_{0}}{N+1} \leq \frac{y_{0}+N y_{0}}{N+1}=y_{0},
\end{aligned}
$$


that is, $x_{0} \leq x_{1} \leq y_{1} \leq y_{0}$. By induction, we have $x_{n} \leq x_{n+1} \leq y_{n+1} \leq y_{n}$. Thus we get that

$$
\theta \leq y_{n}-x_{n}=\frac{A\left(u, y_{n-1}\right)+N y_{n-1}-A\left(u, x_{n-1}\right)-N x_{n-1}}{N+1} \leq \frac{N}{N+1}\left(y_{n-1}-x_{n-1}\right) .
$$

Since $P$ is a normal cone and by induction, we get that

$$
\left\|y_{n}-x_{n}\right\| \leq C\left(\frac{N}{N+1}\right)^{n}\left\|y_{0}-x_{0}\right\|,
$$

where $C$ is the normal constant of $P$.

Moreover (for any natural number $p$ ),

$$
\begin{aligned}
& x_{n+p}-x_{n} \leq y_{n+p}-x_{n} \leq y_{n}-x_{n}, \\
& \left\|x_{n+p}-x_{n}\right\| \leq C\left\|y_{n}-x_{n}\right\| \leq C^{2}\left(\frac{N}{N+1}\right)^{n}\left\|y_{0}-x_{0}\right\|,
\end{aligned}
$$

which implies that $x_{n}$ is a Cauchy sequence. Noticing $E$ is complete, $x_{n}$ converges to some element, we denote it by $T(u)$. Equation (3.1) implies that $y_{n}$ also converges to $T(u)$. By

$$
\begin{aligned}
& x_{n} \leq T(u) \leq y_{n}, \\
& x_{n+1}=B\left(u, x_{n}\right) \leq B(u, T(u)) \leq B\left(u, y_{n}\right) \leq y_{n+1},
\end{aligned}
$$

using the normality of the cone $P$, we can conclude that $x_{n}, y_{n}$ also converge to $B(u, T(u))$. Thus $B(u, T(u))=T(u)$.

If $B(u, x)=x$, then we have for any integer $n, x_{n} \leq x \leq y_{n}$, which implies by taking the limit that $x=T(u)$, i.e., $T(u)$ is the unique fixed point of $A(u, \cdot)$ in $\left[A\left(u_{0}, v_{0}\right), A\left(v_{0}, u_{0}\right)\right]$.

Step 2. We prove that $T(u)$ is increasing in $u$.

If $u, u^{\prime} \in\left[u_{0}, v_{0}\right], u \leq u^{\prime}$, then we let $x_{0}=x_{0}^{\prime}=A\left(u_{0}, v_{0}\right)$ in Step 1 . Since $B(u, v)$ is increasing in both variables, we have

$$
x_{1}=B\left(u, x_{0}\right) \leq B\left(u^{\prime}, x_{0}^{\prime}\right)=x_{1}^{\prime} .
$$

By induction we know $x_{n} \leq x_{n}^{\prime}$. Taking the limit, we have $T(u) \leq T\left(u^{\prime}\right)$.

Step 3. We prove that $T(\cdot)$ has a unique fixed point in $\left[u_{0}, v_{0}\right]$.

Let $u_{n+1}=T\left(u_{n}\right), v_{n+1}=T\left(v_{n}\right)$, we have $u_{0} \leq u_{1}, v_{1} \leq v_{0}$. Thus, by conclusion of Step 2, we get $u_{n} \leq u_{n+1}, v_{n+1} \leq v_{n}$, and $u_{n} \leq v_{n}$. By condition (i), we have

$$
\begin{aligned}
u_{1} & =A\left(u_{0}, T\left(u_{0}\right)\right) \\
& \geq A\left(r_{0} v_{0}, T\left(u_{0}\right)\right) \\
& \geq \varphi\left(r_{0}, v_{0}\right) A\left(v_{0}, T\left(u_{0}\right)\right) \\
& \geq \varphi\left(r_{0}, v_{0}\right) A\left(v_{0}, T\left(v_{0}\right)\right) \\
& =\varphi\left(r_{0}, v_{0}\right) v_{1},
\end{aligned}
$$

that is

$$
u_{1} \geq \varphi\left(r_{0}, v_{0}\right) v_{1}
$$


Let $r_{n}=\varphi\left(r_{n-1}, v_{n-1}\right), n=1,2, \ldots$. By induction, we know

$$
u_{n} \geq r_{n} v_{n}, \quad n=1,2, \ldots
$$

Obviously, the sequence $\left\{r_{n}\right\}$ is increasing with $\left\{r_{n}\right\} \subset(0,1]$. Suppose $r_{n} \rightarrow r(n \rightarrow \infty)$, then $r=1$. Otherwise, we have $0<r<1$. Thus, by condition (iii), we have

$$
r_{n}=\varphi\left(r_{n-1}, v_{n-1}\right) \geq \varphi\left(r_{n-1}, w_{0}\right) .
$$

Let $n \rightarrow \infty$ in (3.6), we have

$$
r \geq \lim _{s \rightarrow r^{-}} \varphi\left(s, w_{0}\right)>r
$$

which is a contradiction. Hence, we have $r=1$.

Therefore, $\forall n, p \geq 1$, we get

$$
\theta \leq v_{n}-u_{n} \leq v_{n}-r_{n} v_{n}=\left(1-r_{n}\right) v_{n} \leq\left(1-r_{n}\right) v_{0}
$$

and

$$
\begin{aligned}
& \theta \leq u_{n+p}-u_{n} \leq v_{n+p}-u_{n} \leq v_{n}-u_{n}, \\
& \theta \leq v_{n}-v_{n+p} \leq v_{n}-u_{n+p} \leq v_{n}-u_{n} .
\end{aligned}
$$

Thus, by the normality of $P$, it is easy to see that $v_{n}-u_{n} \rightarrow 0(n \rightarrow \infty)$, and hence $\left\{u_{n}\right\},\left\{v_{n}\right\}$ are Cauchy sequences. Therefore, there exist $u^{*}, v^{*}$ such that $u_{n} \rightarrow u^{*}, v_{n} \rightarrow v^{*}(n \rightarrow \infty)$ and $u^{*}=v^{*}$. Write $x^{*}=u^{*}=v^{*}$.

Now we show that $T\left(x^{*}\right)=x^{*}$. It is easy to see that

$$
T\left(x^{*}\right) \geq T\left(u_{n}\right)=u_{n+1} \rightarrow x^{*},
$$

and so $T\left(x^{*}\right) \geq x^{*}$. On the other hand, we have

$$
T\left(x^{*}\right) \leq T\left(v_{n}\right)=v_{n+1} \rightarrow x^{*},
$$

so we obtain $T\left(x^{*}\right) \leq x^{*}$. Hence $T\left(x^{*}\right)=x^{*}$.

The proof of the uniqueness is the same as above in Step 1. Finally, from Step 1 we get that $x^{*}$ is the fixed point of $A$ in $\left[u_{0}, v_{0}\right]$ such that $T\left(x^{*}\right)=x^{*}$ and $A\left(x^{*}, x^{*}\right)=x^{*}$.

By the construction we can see that it is unique. If $\bar{x}$ satisfies $A(\bar{x}, \bar{x})=\bar{x}$. By Step $1, T(\bar{x})$ is the unique fixed point of $A(\bar{x}, \cdot)$, thus $T(\bar{x})=\bar{x}$. By the uniqueness of the fixed point of $T(\cdot)$, we get $\bar{x}=x^{*}$. This ends the proof of Theorem 3.1.

Remark 3.1 In fact, by Theorem 2.3 in [30] and Theorem 1.1 in [29], we can know that condition $\lim _{s \rightarrow t^{-}} \varphi\left(s, w_{0}\right)>t$ in Theorem 3.1 can be deleted, while the conclusions remain the same. 
Theorem 3.2 Let E be a real Banach space and P be a cone of E. Let $u_{0}, v_{0} \in E$ with $u_{0} \leq v_{0}$ and $A:\left[u_{0}, v_{0}\right] \times P \rightarrow P$ be a generalized condensing and mixed monotone operator. For fixed $v \in P, A(\cdot, v):\left[u_{0}, v_{0}\right] \rightarrow P$ is a $\varphi$-concave operator. Suppose that conditions (i)-(iv) of Theorem 3.1 hold. Then $A$ has exactly one fixed point $x^{*}$ in $\left[u_{0}, v_{0}\right]$.

Proof For fixed $u \in\left[u_{0}, v_{0}\right]$, let $S=\left\{x_{n} \mid n=0,1,2, \ldots\right\}$. We show that $S \subset \operatorname{co}\left\{x_{0}, A(S)\right\}$. It is easy to see that $x_{0} \in \operatorname{co}\left\{x_{0}, A(S)\right\}$. If $x_{k} \in \operatorname{co}\left\{x_{0}, A(S)\right\}(k \geq 0)$, then for fixed $u \in\left[u_{0}, v_{0}\right]$,

$$
x_{k+1}=\frac{A\left(u, x_{k}\right)+N x_{k}}{N+1}=\frac{A\left(u, x_{k}\right)}{N+1}+\left(1-\frac{1}{N+1}\right) x_{k} .
$$

Noticing that $A\left(u, x_{k}\right), x_{k} \in \operatorname{co}\left\{x_{0}, A(S)\right\}$, we have that $x_{k+1} \in \operatorname{co}\left\{x_{0}, A(S)\right\}$. By induction, we have that $S \subset \operatorname{co}\left\{x_{0}, A(S)\right\}$. It follows from Lemma 2.1 that

$$
\alpha(S) \leq \alpha\left(\operatorname{co}\left\{x_{0}, A(S)\right\}\right)=\alpha(A(S))<\alpha(S) .
$$

This is a contradiction. Consequently, we find that $\alpha(S)=0$. In view of Lemma 2.1, we have that $\bar{S}$ is a compact set in $E$. This implies that there exists a subsequence $\left\{x_{n_{i}}\right\}$ of $\left\{x_{n}\right\}$, which converges to $x^{*}$ in $E$.

Now, we prove that $\left\{x_{n}\right\}$ itself also converges to $x^{*}$. If not, there exists another subsequence $\left\{x_{n_{j}}\right\}$ of $\left\{x_{n}\right\}$, which converges to another point $x_{*} \neq x^{*}\left(x_{*} \in E\right)$. Thus, for any fixed $\left\{x_{n_{i_{0}}}\right\}$, and if $n_{j}$ is large enough, then $x_{n_{i_{0}}} \leq x_{n_{j}}$. Let $n_{j} \rightarrow \infty$, we get that $x_{n_{i_{0}}} \leq x_{*}$. Since $x_{n_{i_{0}}}$ is any fixed element of $\left\{x_{n_{i}}\right\}$, then for any element $x_{n_{i}}$ of $\left\{x_{n_{i}}\right\}$, we have that $x_{n_{i}} \leq x_{*}$. Let $n_{i} \rightarrow \infty$, we get that $x^{*} \leq x_{*}$. Similarly, we can prove that $x_{*} \leq x^{*}$. Thus, $x_{*}=x^{*}$. This is a contradiction. Therefore, $x_{n} \rightarrow x^{*}$. In the same method, we can know that there exists $y^{*} \in E$ such that $y_{n} \rightarrow y^{*}$.

Since $\theta \leq y_{n}-x_{n} \leq\left(\frac{N}{N+1}\right)^{n}\left(y_{0}-x_{0}\right), n=1,2,3, \ldots$ Let $n \rightarrow \infty$, we have that $y^{*}=x^{*}$.

In the following, we show that $x^{*}$ is the fixed point of $A$. In fact, if $m \geq 1$, for any fixed integer $n, x_{n} \leq x_{n+m} \leq y_{n}$. Let $m \rightarrow \infty$, we have that $x_{n} \leq x^{*} \leq y_{n}$. Thus,

$$
\begin{aligned}
A\left(u, x^{*}\right) & =A\left(u, x^{*}\right)-A\left(u, x_{n-1}\right)+A\left(u, x_{n-1}\right) \\
& \geq-N\left(x^{*}-x_{n-1}\right)+A\left(u, x_{n-1}\right) \\
& =-N x^{*}+N x_{n-1}+A\left(u, x_{n-1}\right) \\
& =-N x^{*}+(N+1) x_{n} \rightarrow x^{*} \quad(n \rightarrow \infty) .
\end{aligned}
$$

On the other hand,

$$
\begin{aligned}
A\left(u, x^{*}\right) & =A\left(u, x^{*}\right)-A\left(u, y_{n-1}\right)+A\left(u, y_{n-1}\right) \\
& \leq-N\left(x^{*}-y_{n-1}\right)+A\left(u, y_{n-1}\right) \\
& =-N x^{*}+N y_{n-1}+A\left(u, y_{n-1}\right) \\
& =-N x^{*}+(N+1) y_{n} \rightarrow x^{*} \quad(n \rightarrow \infty) .
\end{aligned}
$$

Therefore, for fixed $u \in\left[u_{0}, v_{0}\right], A\left(u, x^{*}\right)=x^{*}$. The rest of the proof is the same as that of Theorem 3.1. 
Corollary 3.1 (see Theorem 2.1 in [32]) Let $P$ be a normal cone of E, and let $A: P \times P \rightarrow P$ be a mixed monotone operator. Suppose that

1. For fixed $v \in P, A(\cdot, v): P \rightarrow P$ is concave; for fixed $u \in P, \exists N>0$ such that $A(u, \cdot): P \rightarrow P, A\left(u, v_{1}\right)-A\left(u, v_{2}\right) \geq-N\left(v_{1}-v_{2}\right), \forall v_{1} \geq v_{2}, v_{1}, v_{2} \in P$.

2. $\exists \bar{v}>\theta, 0<c \leq 1$ such that $\theta<A(\bar{v}, \theta) \leq \bar{v}$ and

$$
A(\theta, \bar{v}) \geq c A(\bar{v}, \theta)
$$

Then $A$ has exactly one fixed point $u^{*}$ in $[\theta, \bar{v}]$ and $A(\theta, \bar{v}) \leq u^{*} \leq A(\bar{v}, \theta)$.

Proof Set

$$
u_{n}=A\left(u_{n-1}, v_{n-1}\right), \quad v_{n}=A\left(v_{n-1}, u_{n-1}\right), \quad n=1,2, \ldots,
$$

$u_{0}=\theta, v_{0}=\bar{v}$, it is easy to show that

$$
\begin{aligned}
& u_{0}<A\left(v_{0}, u_{0}\right) \leq v_{0}, \quad A\left(u_{0}, v_{0}\right) \geq c A\left(v_{0}, u_{0}\right) \\
& u_{1} \geq c v_{1}, \quad u_{1}=A\left(u_{0}, v_{0}\right) \leq A\left(u_{1}, v_{1}\right) \\
& A\left(v_{1}, u_{1}\right) \leq v_{1}=A\left(v_{0}, u_{0}\right)
\end{aligned}
$$

Now let us prove that $A(\cdot, v):\left[u_{1}, v_{1}\right] \rightarrow P$ is a $\varphi$-concave operator for fixed $v \in\left[u_{1}, v_{1}\right]$. It suffices to show $A(\cdot, v):\left[u_{0}, v_{0}\right] \rightarrow P$ is a $\varphi$-concave operator for fixed $v \in\left[u_{0}, v_{0}\right]$.

For each $u \in\left[u_{0}, v_{0}\right], t \in(0,1)$, we see

$$
\begin{aligned}
A(t u, v) & =A(t u+(1-t) \theta, v) \\
& \geq t A(u, v)+(1-t) A(\theta, v) \\
& \geq t A(u, v)+(1-t) A\left(\theta, v_{0}\right) \\
& \geq t A(u, v)+c(1-t) A\left(v_{0}, u_{0}\right) \\
& \geq t A(u, v)+c(1-t) A(u, v) .
\end{aligned}
$$

Set $\varphi(t, x)=t+c(1-t)$, then $\varphi:(0,1] \times\left[u_{0}, v_{0}\right] \rightarrow(0,1], \varphi(t, x)>t, \forall t \in(0,1), \lim _{s \rightarrow t^{-}} \varphi(s$, $\left.w_{0}\right)=t+c(1-t)>t$.

So, by Theorem 3.1, we see that $A$ has exactly one fixed point $x^{*}$ in $\left[u_{1}, v_{1}\right]$.

Similarly, if $P$ is a solid cone, we have the following corollary.

Corollary 3.2 (see Theorem 2.3 in [32]) Let $P$ be a normal solid cone of E, and let A : $P^{\circ} \times P^{\circ} \rightarrow P^{\circ}$ be a mixed monotone operator. Suppose that

1. For fixed $v \in P^{\circ}, A(\cdot, v): P^{\circ} \rightarrow P^{\circ}$ is concave; for fixed $u \in P^{\circ}, \exists N>0$ such that $A(u, \cdot): P^{\circ} \rightarrow P^{\circ}, A\left(u, v_{1}\right)-A\left(u, v_{2}\right) \geq-N\left(v_{1}-v_{2}\right), \forall v_{1} \geq v_{2}, v_{1}, v_{2} \in P^{\circ}$.

2. $\exists u_{0} \in P^{\circ}, v_{0} \in P^{\circ}$ such that $u_{0} \leq v_{0}, u_{0} \ll A\left(u_{0}, v_{0}\right), A\left(v_{0}, u_{0}\right) \leq v_{0}$.

Then $A$ has exactly one fixed point in $\left[u_{0}, v_{0}\right]$.

Proof It is easy to show that there exists a real number $r_{0}>0$ such that $u_{0} \geq r_{0} v_{0}$ since $u_{0}, v_{0} \in P^{\circ}$, which completes the proof of Corollary 3.2 by means of Corollary 3.1. 
Corollary 3.3 (see Remark 2.4 in [32]) Let $P$ be a normal solid cone of E, and let $A: P^{\circ} \times$ $P \rightarrow P^{\circ}$ be a mixed monotone operator. Suppose that

1. For fixed $v \in P, A(\cdot, v): P^{\circ} \rightarrow P^{\circ}$ is $\alpha$-concave; for fixed $u \in P, \exists N>0$ such that $A(u, \cdot): P \rightarrow P, A\left(u, v_{1}\right)-A\left(u, v_{2}\right) \geq-N\left(v_{1}-v_{2}\right), \forall v_{1} \geq v_{2}, v_{1}, v_{2} \in P$.

2. $\exists u_{0} \in P^{\circ}, v_{0} \in P^{\circ}$ such that $u_{0} \leq v_{0}, u_{0} \ll A\left(u_{0}, v_{0}\right), A\left(v_{0}, u_{0}\right) \leq v_{0}$.

Then $A$ has exactly one fixed point in $\left[u_{0}, v_{0}\right]$.

Proof Take $\varphi(t, x)=t^{\alpha}, \forall t \in(0,1)$. Thus, by Corollary 3.2, we easily see that the conclusions of Corollary 3.3 hold.

Corollary 3.4 (see Theorem 2.2 in [11] or Theorem 2.1 in [10]) Assume that the operator A satisfies the following conditions:

$\left(\mathrm{H}_{1}\right) A: P_{h} \rightarrow P_{h}$ is increasing in $P_{h}$;

$\left(\mathrm{H}_{2}\right)$ for $\forall x \in P_{h}$ and $t \in(0,1)$, there exists $\alpha(t) \in(0,1)$ such that

$$
A(t x) \geq t^{\alpha(t)} A x
$$

Then A has a unique solution in $P_{h}$.

Proof If $A(u, v)$ is independent of $v$, then take $\varphi(t, x)=t^{\alpha(t)}, \forall t \in(0,1)$. It is easy to check that conditions (i), (iii) in Theorem 3.1 hold. By Lemma 2.1 in [8], assume that the operator $A$ satisfies conditions $\left(\mathrm{H}_{1}\right)$ and $\left(\mathrm{H}_{2}\right)$, we can know that there are $u_{0}, v_{0} \in P_{h}$ such that $u_{0}<$ $v_{0}, u_{0} \leq A u_{0} \leq A v_{0} \leq v_{0}$. Thus, condition (ii) in Theorem 3.1 holds. Therefore, it follows from Theorem 3.1 that the conclusions of Corollary 3.4 hold.

Corollary 3.5 (see Theorem 2.6 in [10]) Assume that the operator A satisfies the following conditions:

$\left(\mathrm{H}_{3}\right) A: P_{h} \rightarrow P_{h}$ is increasing in $P_{h}$

$\left(\mathrm{H}_{4}\right)$

$$
A(t x) \geq t^{\alpha(t, x)} A x, \quad \forall x \in P_{h}, t \in(0,1),
$$

where $\alpha:(0,1) \times P_{h} \rightarrow(0,1)$ is increasing in $x$ for fixed $t \in(0,1)$;

$\left(\mathrm{H}_{5}\right)$ there exists $t_{0} \in(0,1)$ such that

$$
t_{0} h \leq A h \leq \frac{1}{t_{0}^{1-\alpha\left(t_{0}, \frac{1}{t_{0}} h\right)}} h .
$$

Then $A$ has a unique solution in $P_{h}$.

Proof By Corollary 3.4, we only need to check condition (ii) in Theorem 3.1. By the proof of Theorem 2.6 in [10], choose $k \in R$ such that $k>\frac{1}{1-\alpha\left(t_{0}\right)}$. Put $u_{0}=t_{0}^{k} h, v_{0}=\frac{1}{t_{0}^{k}} h$, set

$$
u_{n}=A u_{n-1}, \quad v_{n}=A v_{n-1}, \quad n=1,2, \ldots,
$$

we have $u_{0}, v_{0} \in P_{h}, u_{0}<v_{0}$, and $u_{0} \leq A u_{0} \leq A v_{0} \leq v_{0}$.

Furthermore, we can easily obtain the following new result. 
Theorem 3.3 Let $P$ be a normal cone of the real Banach space $E, e>\theta$ and $u_{0}, v_{0} \in P$ with $u_{0} \leq v_{0}$, and let $A: P \times P \rightarrow P$ be a mixed monotone operator. Suppose that

(i) there exists a real positive number $r_{0}$ such that $u_{0} \geq r_{0} v_{0}$;

(ii) $u_{0} \leq A\left(u_{0}, v_{0}\right), A\left(v_{0}, u_{0}\right) \leq v_{0}$;

(iii) for fixed $v, A(\cdot, v): P \rightarrow P$ is e-concave with its characteristic function, $\eta(t, x)$ is monotone in $x$ and continuous in $t$ from left;

(iv) for fixed $u \in P, \exists N>0$ such that

$$
A(u, \cdot): P \rightarrow P, \quad A\left(u, v_{1}\right)-A\left(u, v_{2}\right) \geq-N\left(v_{1}-v_{2}\right), \quad \forall v_{1} \geq v_{2}, v_{1}, v_{2} \in P .
$$

Then $A$ has exactly one fixed point $x^{*}$ in $\left[u_{0}, v_{0}\right]$.

Corollary 3.6 (see Theorem 2.5 in [11]) Assume that the operator A satisfies the following conditions:

$\left(\mathrm{H}_{6}\right) A: P_{h} \rightarrow P_{h}$ is increasing in $P_{h}$;

$\left(\mathrm{H}_{7}\right)$ for each $x \in P_{h}$ and $t \in(0,1)$, there exists $\eta(t) \in(0,1)$ such that

$$
A(t x) \geq t(1+\eta(t)) A x
$$

Then $A$ has a unique solution in $P_{h}$.

Proof Set $\eta(t, x)=\eta(t)$, by Corollary 3.4 and Theorem 3.3, we can know that the conclusions of Corollary 3.6 hold.

\section{Application}

In this section, we present an example to explain our results.

Example 4.1 Consider the following nonlinear integral equation:

$$
x(t)=(A x)(t)=\int_{R^{N}} K(t, s)\left[x^{\frac{1}{2}}(s)+x(s)+x^{-\frac{1}{3}}(s)\right] d s .
$$

Conclusion 4.1 Suppose that $K: R^{N} \times R^{N} \rightarrow R^{1}$ is nonnegative and continuous with

$$
\frac{1}{111} \leq \int_{R^{N}} K(t, s) d s \leq \frac{1}{6} .
$$

Then Eq. (4.1) has a unique positive solution $x^{*}(t)$ satisfying $10^{-2} \leq x^{*}(t) \leq 1$.

Proof We use Theorem 3.3 to prove Conclusion 4.1. Let $E=C_{B}\left(R^{N}\right)$ denote the set of all bounded continuous functions on $R^{N}$; we define $\|x\|=\sup _{t \in R^{N}}|x(t)|$, and then $E$ is a real Banach space. Let $P=C_{B}^{+}\left(R^{N}\right)$ denote the set of all nonnegative functions of $C_{B}\left(R^{N}\right)$. Then $P$ is a normal cone of $E$. Obviously, Eq. (4.1) can be written in the form $x=A(x, x)$, where

$$
\begin{aligned}
& A(x, y)=A_{1}(x)+A_{2}(y), \\
& A_{1}(x)=\int_{R^{N}} K(t, s)\left[x^{\frac{1}{2}}(s)+x(s)\right] d s, \\
& A_{2}(y)=\int_{R^{N}} K(t, s) y^{-\frac{1}{3}}(s) d s .
\end{aligned}
$$


Now, let us show that the operator $A$ satisfies all the conditions in Theorem 3.3. In fact, set $u_{0}=10^{-2}, v_{0}=1$. It is easy to see that $A: P \times P \rightarrow P$ is a mixed monotone operator. It is obvious that $u_{0}, v_{0} \in P, u_{0}<v_{0}$ and there exists a real number $\epsilon_{0}>0$ such that $u_{0} \geq \epsilon_{0} v_{0}$. By (4.2) we can easily get

$$
A\left(u_{0}, v_{0}\right)=\int_{R^{N}} K(t, s)\left(10^{-1}+10^{-2}+1\right) d s \geq 10^{-2}=u_{0},
$$

and

$$
A\left(v_{0}, u_{0}\right)=\int_{R^{N}} K(t, s)\left(1+1+10^{\frac{2}{3}}\right) d s \leq 1=v_{0} .
$$

For fixed $y, \forall t \in(0,1), \exists \eta=\eta(t, x)=\frac{(\sqrt{t}-t) \sqrt{x}}{t(\sqrt{x}+x)}>0$ such that

$$
A(t x, y) \geq(1+\eta) t A(x, y)
$$

where $\eta(t, x)$ is decreasing in $x$ and continuous in $t$ from left.

For fixed $x, \exists N=\frac{500}{9}$ such that

$$
A\left(x, v_{1}\right)-A\left(x, v_{2}\right) \geq-\frac{500}{9}\left(v_{1}-v_{2}\right), \quad \forall v_{1} \geq v_{2}, v_{1}, v_{2} \in\left[10^{-2}, 1\right]
$$

Therefore, we see Conclusion 4.1 holds by means of Theorem 3.3.

\section{Competing interests}

The author declares that they have no competing interests.

\section{Acknowledgements}

The author was supported financially by the National Natural Science Foundation of China, Tianyuan Foundation (11226119), the Scientific and Technological Innovation Programs of Higher Education Institutions in Shanxi, the Youth Science Foundation of Shanxi Province (2013021002-1), and Shandong Provincial Natural Science Foundation, China (ZR2012AQ024).

Received: 4 August 2013 Accepted: 9 October 2013 Published: 08 Nov 2013

\section{References}

1. Amann, H: Fixed point equations and nonlinear eigenvalue problems in ordered Banach spaces. SIAM Rev. 18, 620-709 (1976)

2. Guo, DJ: Nonlinear Functional Analysis. Shandong Science and Technology Press, Jinan (2001) (in Chinese)

3. Guo, DJ, Lakshmikantham, V: Nonlinear Problems in Abstract Cones. Academic Press, San Diego (1988)

4. Krasnoselskii, MA: Positive Solutions of Operator Equations. Noordhoff, Groningen (1964)

5. Krasnoselskii, MA, Zabreiko, PP: Geometrical Methods of Nonlinear Analysis. Springer, Berlin (1984)

6. Liang, Z, Lian, X, Zhang, M: A class of concave operators with applications. Nonlinear Anal. 68, 2507-2515 (2008)

7. Potter, AJB: Applications of Hilbert projective metric to certain class of nonhomogeneous operators. Q. J. Math. 28, 93-99 (1977)

8. Xu, SY, Jia, BG: Fixed-point theorems of $\phi$-concave $(-\psi)$ convex mixed monotone operators and applications. J. Math. Anal. Appl. 295, 645-657 (2004)

9. Xu, SY, Zeng, CY, Zhu, CX: Existence and uniqueness for the fixed points of $\phi$-concave $(-\psi)$ convex mixed monotone operators and applications. Acta Math. Sin. 48, 1055-1064 (2005) (in Chinese)

10. Zhai, CB, Wang, WX, Zhang, LL: Generalizations for a class of concave and convex operators. Acta Math. Sin. 51, 529-540 (2008) (in Chinese)

11. Zhai, CB, Yang, C, Guo, CM: Positive solutions of operator equations on ordered Banach spaces and applications Comput. Math. Appl. 56, 3150-3156 (2008)

12. Zhao, ZQ, Du, X: Fixed points of generalized e-concave (generalized e-convex) operators and their applications. J. Math. Anal. Appl. 334, 1426-1438 (2007)

13. Karapinar, E, Luong, NV, Thuan, NX: Coupled coincidence points for mixed monotone operators in partially ordered metric spaces. Arab. J. Math. 1, 329-339 (2012)

14. Liang, Z, Wang, W, Li, S: On concave operators. Acta Math. Sin. New Ser. 22, 577-582 (2006) 
15. Li, FY, Liang, ZD: Fixed point of $\varphi$-concave(- $\varphi$-convex) operator and application. J. Syst. Sci. Math. Sci. 14(4), 355-360 (1994)

16. Guo, DJ, Lakshmikantham, V: Coupled fixed points of nonlinear operators with applications. Nonlinear Anal. TMA 11(5), 623-632 (1987)

17. Abbas, M, Ali, B, Sintunavarat, W, Kumam, P: Tripled fixed point and tripled coincidence point theorems in intuitionistic fuzzy normed spaces. Fixed Point Theory Appl. 2012, 187 (2012)

18. Abbas, M, Sintunavarat, W, Kumam, P: Coupled fixed point of generalized contractive mappings on partially ordered G-metric spaces. Fixed Point Theory Appl. 2012, 31 (2012)

19. Aydi, H, Abbas, $M$, Sintunavarat, W, Kumam, P: Tripled fixed point of $W$-compatible mappings in abstract metric spaces. Fixed Point Theory Appl. 2012, 134 (2012)

20. Gnana Bhaskar, T, Lakshmikantham, V: Fixed point theorems in partially ordered metric spaces and applications. Nonlinear Anal. TMA 65(7), 1379-1393 (2006)

21. Chandok, S, Sintunavarat, W, Kumam, P: Some coupled common fixed points for a pair of mappings in partially ordered G-metric spaces. Math. Sci. 7, 24 (2013)

22. Drici, Z, McRae, FA, Vasundhara Devi, J: Fixed point theorems for mixed monotone operators with PPF dependence. Nonlinear Anal. TMA 69, 632-636 (2008)

23. Guo, DJ: Existence and uniqueness of positive fixed point for mixed monotone operators and applications. Appl. Anal. 46, 91-100 (1992)

24. Guo, DJ: Fixed points for mixed monotone operators with application. Appl. Anal. 34, $215-224$ (1988)

25. Harjani, J, López, B, Sadarangani, K: Fixed point theorems for mixed monotone operators and applications to integral equations. Nonlinear Anal. TMA 74, 1749-1760 (2011)

26. Karapinar, E, Kumam, P, Sintunavarat, W: Coupled fixed point theorems in cone metric spaces with a c-distance and applications. Fixed Point Theory Appl. 2012, 194 (2012)

27. $\mathrm{Wu}, \mathrm{YS}, \mathrm{Li}, \mathrm{GZ}$ : Existence and uniqueness theorems of fixed points for mixed monotone operators and their applications. Acta Math. Sin. (Chin. Ser.) 46(1), 161-166 (2003) (in Chinese)

28. Zhai, CB: Fixed point theorems for a class of mixed monotone operators with convexity. Fixed Point Theory Appl. 2013, 119 (2013)

29. Zhai, CB, Zhang, LL: New fixed point theorems for mixed monotone operators and local existence-uniqueness of positive solutions for nonlinear boundary value problems. J. Math. Anal. Appl. 382, 594-614 (2011)

30. Zhang, ZT: Fixed point theorems of mixed monotone operators and its applications. Acta Math. Sin. 41(6), $1121-1126$ (1998) (in Chinese)

31. Zhang, ZT: New fixed point theorems of mixed monotone operators and applications. J. Math. Anal. Appl. 204(1) 307-319 (1996)

32. Zhang, ZT, Wang, K: On fixed point theorems of mixed monotone operators and applications. Nonlinear Anal. TMA 70, 3279-3284 (2009)

33. Zhao, ZQ: Uniqueness and existence of fixed points on some mixed monotone mappings in ordered linear spaces. J. Syst. Sci. Math. Sci. 19(2), 217-224 (1999)

34. Sintunavarat, W, Kumam, P: Coupled coincidence and coupled common fixed point theorems in partially ordered metric spaces. Thai J. Math. 10(3), 551-563 (2012)

35. Sintunavarat, W, Cho, YJ, Kumam, P: Coupled fixed-point theorems for contraction mapping induced by cone ball-metric in partially ordered spaces. Fixed Point Theory Appl. 2012, 128 (2012

36. Guo, YX: Iterative solution on a class of nonlinear operator equation. Acta Anal. Funct. Appl. 1, $45-50$ (1999)

37. Sun, JX: Iterative solution of nonlinear operator equation (II). J. Shandong Univ. 3, 281-288 (1992) (in Chinese)

10.1186/1687-1812-2013-274

Cite this article as: Sang: A class of $\varphi$-concave operators and applications. Fixed Point Theory and Applications 2013, 2013:274

\section{Submit your manuscript to a SpringerOpen ${ }^{\ominus}$ journal and benefit from:}

- Convenient online submission

- Rigorous peer review

- Immediate publication on acceptance

- Open access: articles freely available online

- High visibility within the field

- Retaining the copyright to your article 